\title{
The Sociodental Approach To Assess Dental Treatment Needs - A Review
}

\author{
${ }^{1}$ Dr. Susanthironanki, ${ }^{2}$ Dr. Suhaskulkarni, ${ }^{3}$ Dr. B.Srikanth Reddy, \\ ${ }^{4}$ Dr. Dolardoshi, ${ }^{5}$ dr. Padma Reddy
}

\begin{abstract}
Health needs assessment is an important component in health care planning. It provides a platform to systemically assess unmet health and health care needs and to collect data required in improving health of the population. The traditional normative need approach for lacking in its objectivity and reliability, for ignoring the concept of quality of life and for disregarding scientific evidence, there is a need to develop appropriate measure of oral health needs which would incorporate the impact of ill-health on daily life, the degree of dysfunction it causes and the perceptions and attitudes of patients which would provide a better division of resources in providing care. This research highlights thesociodental approach, which is conceptually appropriate as it correspond to broader concepts of health and needs. This approach conforms to the modern, theoretical, multifactorial approach for the assessment of oral health care needs.
\end{abstract}

Keywords: Need, Sociodental, Demand

\section{Introduction}

Needs can be viewed from different perspectives; a sociological, an epidemiological and an economic point of view. A sociological standpoint is exemplified by Bradshaw (1972) ${ }^{1}$ who classified needs in terms of i) normative need (professionally defined needs), ii) felt need (individual perception of want), iii) expressed need (vocalized needs or demand for service) and iv) comparative need (comparison of needs among different people on the health care that they received). This taxonomy demonstrates that there are no absolute measures of needs (Thayer 1973). Bradshaw's definition has been a useful framework for policy making as it categorizes the different factors that might influence needs. Needs measured using an epidemiological approach is defined in terms of the morbidity and mortality status or in other words the amount of ill health or disease in the population. It focused on normative or professionally defined needs which may be expressed in terms of items of health care, or need equivalents such as time, cost or workforce requirements ${ }^{3}$. This approach is similar with the humanitarian approach of health care which implies that action must be taken when disease is detected ${ }^{4}$. There is no resource allocation rule within this approach; assessment of needs is made with the assumption that resources will be available to satisfy them. The economists' approach to health care defines needs within the context of cost-effectiveness and supply and demand ${ }^{5}$. Health economics is a branch of economics concerned with issues related to scarcity in the allocation of health and health care. Health needs should only be identified when there is an effective intervention available to improve the health of those in needs ${ }^{6}$. Black $(1994)^{7}$ stressed that although meeting health needs is a moral or ethical question, it is not possible to meet all needs as there are limits to the resources available. Mooney $(1994)^{8}$ concluded that if health care resources are to be allocated on the basis of 'need' or on the capacity of an 'ability to benefit', therefore health needs assessment must inevitably result in some form of rationing. The difference between 'realistic' and 'humanitarian' approaches is that the starting point of the former is identifying resources that are available to meet the needs, while for the latter it starts with identification of need ${ }^{4}$.

Health needs assessment is an important component in health care planning. It provides a platform to systemically assess unmet health and health care needs and to collect data required in improving health of the population. Health gains can therefore be achieved by reallocating resources as a result of identifying ${ }^{9}$ :

- Non-recipients of beneficial health care interventions (that is unmet need)

- Recipients of ineffective health care (and releasing resources for unmet need)

- Recipients of inappropriate health care (for whom the outcomes could be improved)

The estimation of need for health care has been a challenging task because of the difficulty of defining health. To accurately define health needs requires the existence of well-defined standards of what constitutes good health status and knowledge of technology that can improve ill health ${ }^{3}$. However the concept of health is multidimensional and complex and the abundance of terms that have been used to describe it such as wellness, well-being, illness or psychosocial impact, has made defining what health is even more complicated ${ }^{10}$. 


\section{The sociodental approach to assessing dental treatment needs.}

The need to incorporate the functional and social dimensions of dental diseases into the assessment of dental health needs was first expressed by Sheiham et al. $(1982)^{11}$ who pointed out that service-related approach to needs assessment has numerous shortcomings. They recommended that a more appropriate measure of oral health needs would incorporate the impact of ill-health on daily life, the degree of dysfunction it causes and the perceptions and attitudes of patients which would provide a better division of resources in providing care. This idea was supported by Kay (1993) ${ }^{12}$ who felt that the measurements of oral health needs which take into account physical, psychological and social aspects will uphold the status of oral health professionals as one that cares for the society beyond the mere palliative aspect.

Maizels et al (1993) ${ }^{13}$ combined oral health status and oral health behaviour in assessing oral health needs and proposed different strategies to different treatment need group based on their propensities level. Adulyanon (1996) ${ }^{14}$ moved the idea of using sociodental measures to assess needs forward by formulating a comprehensive needs assessment model that includes contemporary concepts of health and which emphasized health gain in assessing needs. The gradual inclusion of oral impact assessments and behavioural propensity into the normative needs assessment provides a systematic approach to identifying priority groups who will benefit from dental interventions. This rational evidence-based patient oriented approach to treatment provision based on the degree of need should serve as an acceptable basis for establishing ethical priorities in health services. This sociodental approach is conceptually appropriate as it correspond to broader concepts of health and needs. This approach conforms to the modern, theoretical, multifactorial approach for the assessment of oral health care needs ${ }^{15}$.

\section{It has four essential components which have been recognized as important in assessing health needs: 1. Clinical Assessment Of Normative Need}

Despite having numerous shortcomings, normative needs is still useful for identifying diseases and impairments. The need for any treatment on an individual can only be estimated after the examination of health professionals by using clinical indices. The data gained can be gathered to produce the prevalence and patterns of diseases in the population. During a diagnostic examination, the dentists are not only looking for existing disease but they are also able to detect any possible signs of future diseases. Hence the type of treatment and prevention measures could be recommended during this assessment.

\section{Subjective Perceptions Of Needs}

This is measured through an oral health related quality of life indicator. Among many of the oral health related quality of life instruments developed, only the Oral Impacts on Daily Performance (OIDP) index constructed by Adulyanon and Sheiham (1997) ${ }^{16}$ is specifically designed for used in treatment needs assessment. It is based on the WHO's International Classification of Impairments, Disabilities and Handicaps which was adopted and modified for dentistry by Locker $(1988)^{17}$. The theoretical model of oral health related quality of life appraisal divides oral health consequences into three levels. The first level refers to oral impairments. The second level 'intermediate impacts', includes the possible earliest negative impacts caused by oral health status which could include pain, discomfort or functional limitation. The third level, which the OIDP index concentrates on, represents impacts on the ability to perform daily activities which composes of physical, psychological and social performance ${ }^{15}$. By focusing on only the third level of health consequences, the OIDP measures only the ultimate impact; that is the behaviour impacts of oral disorders and the extent to which the ability to perform physical, psychological and social performances is compromised ${ }^{18}$. This avoids the double scoring of the same impacts on different levels and resolves the problematic assessment of subjective feelings by measuring the behavioural translation ${ }^{19}$. This has made the index concise and yet comprehensive as it covers the main dental consequences. Assessing only the impact one has from the mouth will not give much information about the type of treatment needed to deal with the condition.

A distinctive feature of the OIDP is that the impacts reported can be attributed to a specific dental condition that is called Condition Specific OIDP (CS-OIDP) ${ }^{15}$. CS-OIDP links the oral impact with the specific oral conditions or diseases as perceived by the patients. For example, a patient that has a normative need for periodontal treatment who reports that having a bad breath has an effect on doing his daily activities which they say is related to their gums can be considered as having an impact related need for a scaling and polishing. Thus this feature enables assessment of the specific treatment need required by the individual, and thereby assists planners in estimating the type of dental workforce required to carry out the treatment needs.

\section{Propensity For Oral Health Behaviours}

A patient's commitment to good oral health is fundamental for a successful outcome in dental treatment. Hence, a measurement of propensity when combined with the professional and lay perceptions should provide a more complete assessment of treatment needs and thereby improve treatment planning ${ }^{20}$. Most 
common oral diseases such as dental caries and periodontal disease are caused by poor oral behavioural which is preventable. To ensure that maximum benefit is gained within available resources, it is vital that treatment is given to those who are more likely to benefit from the treatment ${ }^{15}$.

\author{
There Are Five Oral Health-Related Behaviours Which Could Influence Oral Health Status And \\ Treatment Outcomes: \\ - The use of fluoride toothpaste \\ - The frequency of tooth brushing \\ - Sugar intake \\ - Pattern of dental attendance \\ - Smoking behavior
}

\title{
4. The Effectiveness Of Treatment
}

The long term success of treatment and the positive health outcomes gained by patients should be one of the main criteria to be considered when recommending interventions to the patients. This can be acquired if evidence-based dental care approach (EBD) is practiced. EBD is defined as 'the integration of systematic assessments of clinically relevant scientific evidence, relating to the patient's oral and medical condition and history, with the dentist's clinical expertise and the patient's treatment needs and preferences ${ }^{21}$. The move toward EBD practice has been recommended as it could identify and reduce the use of any treatment shown to be unnecessary or ineffective and release resources for more appropriate and effective uses. The Oral Health Group of the Cochrane Collaboration helps dentist to make well-informed decision regarding oral health care by producing and publishing systematic reviews on the prevention, treatment and rehabilitation of dental disorders.

\section{Integration Of Components Into The Sociodental System For Assessing Dental Treatment Needs}

The sociodental system for needs assessment involves a gradual integration process of the aforementioned essential components (normative needs, subjective perceptions, propensity behaviour and evidence-based treatment). The needs assessment pathway which involves three levels is shown in Figure 1. The first level is the assessment of needs by the professionals based on the best available evidence of the natural history of oral diseases. People with life threatening conditions, such as oral cancer or precancerous lesion, or with chronic progressive conditions such as dentinal caries, will be only evaluated at this level and need not go through subjective assessment. This is because these oral disorders seldom give any symptoms that impact a person quality of life at the early stage of the lesions. Delaying the treatment might deteriorate the conditions. Hence, those with these oral conditions should receive treatment regardless of whether they have any oral impact arising from it. However the type of treatment they receive will depend on their propensity for dental treatments based on their oral health behaviours. On the other hand, individuals with non-life threatening and non-progressive oral conditions will be further assessed at the second level.

The second level of need assessment involves the integration of the normative need (NN) with the reported oral impacts assessed through the OIDP index, which results in 'Impact-related need' (IRN). This level will determine whether the individuals will require the intervention based on their reported disability and handicaps resulting from oral problems. Those whose oral problems do not provoke any impacts on their daily life might not receive the priority in getting dental treatment. A decision on their dental needs should be made by evaluating the level of impacts, the type of treatment available as well as ethical considerations.

The third level takes into the consideration the individuals behavioural propensities for dental treatment and integrates this with the previous IRN, resulting in 'Propensity-related need' (PRN). The oral health behaviour considered depends on the type of treatment that is provided. For example, for restorative treatment, tooth brushing, usage of fluoride and sugar intake should be assessed. Those with high propensities will receive the initially planned dental treatment, those without are considered as having a need to change their behaviour through dental health education / oral health promotion in order to maximise their oral health gain for future dental treatment.

\section{In Summary There Are Two Models Involved In The Sociodental System:}

The first model namely Dental Needs for Life-threatening and Progressive oral conditions (DNLP) is, as the name implies, used for emergency or life threatening and progressive oral conditions and is used for assessments of restorative treatments and extraction needs. In the DNLP model only the Normative Need (NN) andPropensity-related need(PRN) is assessed. The second model is called the Basic Model of Dental Needs (BMDN). This model is for dental conditions other than those stated in the DNLP model and is used in assessing periodontal and prosthodontic treatment needs. It assesses three level of needs; NN, Impact-related need(IRN) and PRN. 
Evidence-based dentistry applies through all the three levels of assessment. The type of treatment offered to the patients must be proper with the local settings, available resources and general needs of the community ${ }^{15}$.

The difference between the oral health needs measured using the normative method and the sociodental approach range between 40 to $90 \%$ when used in children, adolescents, adults and older people ${ }^{16,22,23,24,25}$.

Although the sociodental approach has been shown to give a more rational estimate of dental treatment need compared to normative approach, it has a number of shortcomings. For the BMDN model, because the oral conditions covered by it are considered to be non-progressive, a positive need for treatment depends on the presence of normative need, a sociodental impact and the positive behavioural propensity. No account is taken of the perceived needs expressed by the individual because it is assumed that if a person does not have a sociodental impact they would not seek treatment. Although people may not report the presence of oral impact, they might feel the need for treatment. There are differences between self-reports of dental problems and perceived need for dental care ${ }^{26,27}$. The sociodental account also did not consider the various factors that would affect the demand and utilization of services. Variables such as income, education, age, cultural and racial demography could influence individual's propensity to use health care services regardless of the presence of impact $^{28,29}$. The stringent criterion for allocating treatment to those without oral impact could therefore result in underestimating the people's wants. It could be worthwhile to consider subjective perceived need for condition specific treatments even when there is no impac t related to that condition.

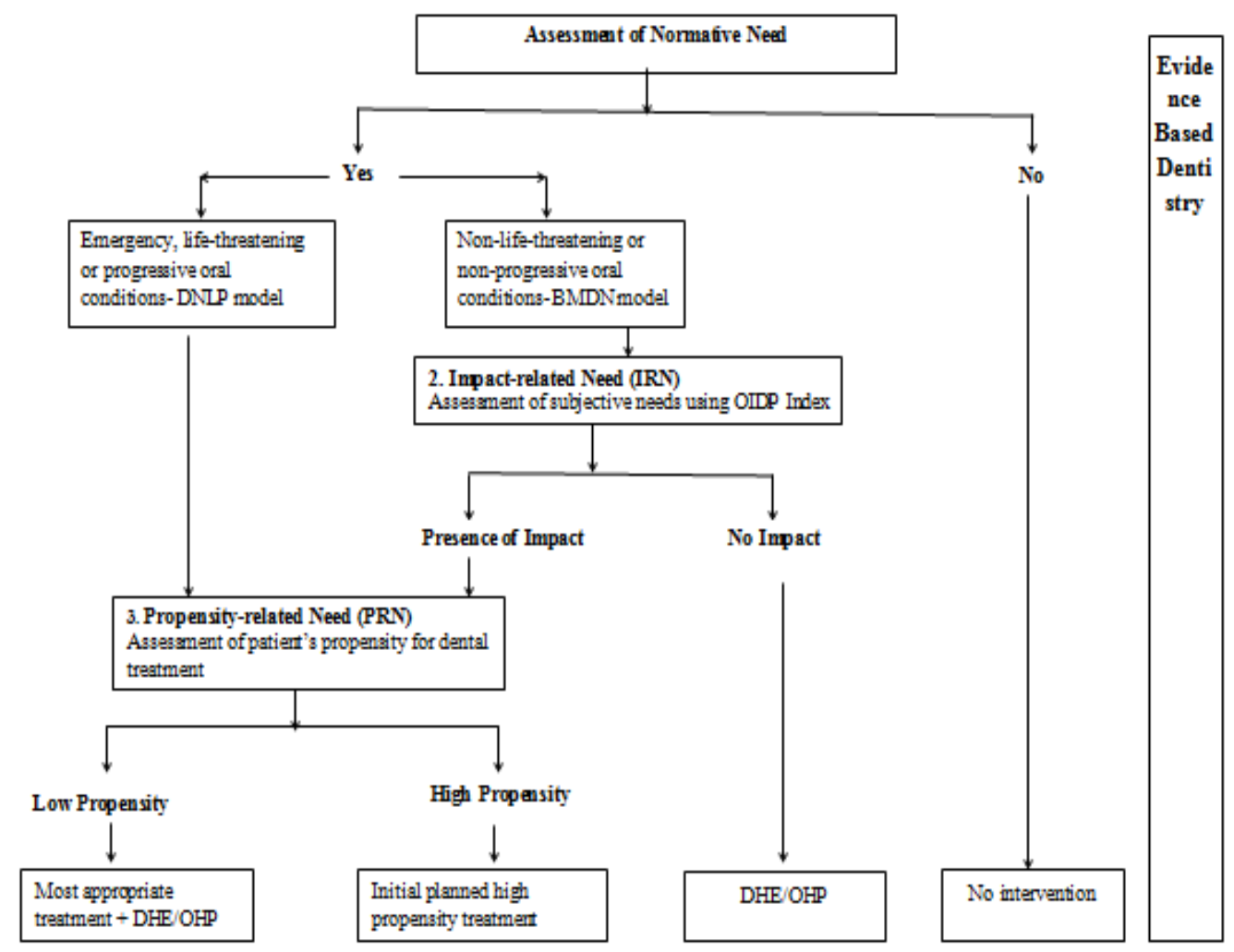

Figure 1: Oral health needs assessment pathway using the sociodental system

(Modified from Adulyanon (1996)

\section{References}

[1]. Bradshaw, J. Taxonomy of social need. In G. McLachlan (ed) Problems and progress in medical care: Essays on current research, seventh series 1972. Oxford: Oxford University Press.

[2]. Thayer, R. (1973). Measuring need in the social services. Soc Policy Admin 1973; 7: 91-105. The Institute for Research of Public Expenditure (2006). Innovation in dental care: recommendations. Leiden, the Netherlands: Secretariat of the Innovation in Dental Care Committee.

[3]. Spencer AJ. The estimation of need for dental care. J Public Health Dent 1980; 40(4): 311-27.

[4]. Acheson RM. The definition and identification of need for health care. J Epidemiol Community Health 1978; 32(1): 10-5.

[5]. Billings JR and Cowley S. Approaches to community needs assessment: a literature review. J AdvNurs 1995; 22(4): 721-30.

[6]. Matthew GK. Measuring need and evaluating services. In. G. McLachlan. Portfolio for health: the role and programme of the DHSS in Health Services Research 1971. Oxford University Press: London.

[7]. Black D. A doctor looks at health economics. Office of Health Economics annual lecture 1994; London: OHE.

[8]. Mooney G. Key issues in health economics.1994; New York City US: Harvester Wheatsheaf. 
[9]. Stevens A and Gillam S. The development of practical approaches to health needs assessment. 1998 In. J. Wright (ed). Health needs assessment in practice. London: BMJ Books.

[10]. Locker D. Concepts of oral health, disease and quality of life. In. G.D. Slade, Measuring oral health and quality of life. University of North Carolina 1997: Chapel Hill, North Carolina.

[11]. Sheiham A, Maizels JE, Cushing, AM. The concept of need in dental care. Int Dent J 1982; 32(3): $265-70$.

[12]. Kay EJ. Patients' needs-more than meets the eye. Br Dent J 1993; 174(6): 212-214.

[13]. Maizels J, Maizels, A., Sheiham, A. Sociodental approach to the identification of dental treatment-need groups. Community Dent Oral Epidemiol 1993; 21(6): 340-346.

[14]. Adulyanon, S. An integrated socio-dental approach to dental treatment estimation: Ph.D. Thesis 1996. University College London.

[15]. Sheiham A and Tsakos G. Oral health needs assessment. In. C. Pine and R. Harris ed. Community Oral Health 2007. 2nd. Quintessence Publishing: Germany.

[16]. Adulyanon S and Sheiham A. Oral impacts on daily performances In: G.D. Slade. Measuring Oral Health and Quality of Life; 1997: Chapel Hill: University of North Carolina.

[17]. Locker D. Measuring oral health: a conceptual framework. Community Dent Health 1988; 5: 3-18.

[18]. Locker D and Allen F. What do measures of 'oral health-related quality of life' measure? Community Dent Oral Epidemiol 2007; 35(6): 401-11.

[19]. Tsakos G. Sociodental impacts related to dental status in older people: A comparative study in Greek and British populations: Ph.D. Thesis; 1998: University College London.

[20]. Sheiham A, Maizels JE and Cushing, AM. The concept of need in dental care. Int Dent J 1982; 32(3): $265-70$.

[21]. ADA. Policy on evidence-based dentistry. Available at: www.ada.org/1754.aspx (Accessed 19 February 2012).

[22]. Srisilapanan P, Korwanich N, Sheiham A. Assessing prosthodontic dental treatment needs in older adults in Thailand: normative vs. sociodental approaches. Spec Care Dentist 2003; 23(4): 131-4.

[23]. Gherunpong S, Tsakos G, Sheiham A. A sociodental approach to assessing dental needs of children: concept and models. Int J Paediatr Dent 2006c: 16(2); 81-88.

[24]. AstromAN and Kida IA. Perceived dental treatment need among older Tanzanian adults - a cross-sectional study. BMC Oral Health 2007; 7: 9 .

[25]. Ryu JI, Tsakos G, Sheiham A. Differences in prosthodontic treatment needs assessments between the standard normative and sociodental approach. Int J Prosthodont 2008; 21(5):425-32.

[26]. Jokovic A and Locker D. Dissatisfaction with oral health status in an older adult population. J Public Health Dent 1997; $57(1)$ : 40-7.

[27]. Heft MW, Gilbert GH, Shelton BJ, and Duncan RP. Relationship of dental status, sociodemographic status, and oral symptoms to perceived need for dental care. Community Dent Oral Epidemiol 2003; 31(5): 351-60.

[28]. Odrich, J. Dental manpower planning: can we ever get it right? J Public Health Pol 1985; 6(4): 539-52.

[29]. Andersen RM. (2008). National health surveys and the behavioural model of health services use. Med Care 2008;47:647-653. 